Revista de Comunicación de la SEECI. (Noviembre de 2014). Año XVII (35), 8-18

ISSN: 1576-3420

http://dx.doi.org/10.15198/seeci.2014.35.8-18

\title{
INVESTIGACIÓN/RESEARCH
}

Recibido: 13/06/2014 Aceptado: 15/09/2014 Publicado: 15/11/2014

\section{FERNANDO II DEL SACRO IMPERIO ROMANO GERMÁNICO}

\section{Andrea Cristina Reglá Carrillo}

Venezuela. Universidad Metropolitana. Caracas (Venezuela)

andreareglac23@gmail.com

\section{María Elena Del Valle Mejías}

Venezuela. Universidad Metropolitana. Caracas (Venezuela)

manedelvalle@gmail.com

RESUMEN: El siguiente artículo desarrolla un aspecto de la vida del emperador del Sacro Imperio Romano Germánico, Fernando II de Habsburgo. Nos interesamos profundamente en este aspecto de su biografía debido a que relata temas tan importantes como el de la Guerra de los Treinta Años. Citando autores excepcionales que enfocaron sus textos en la mencionada Guerra, recopilamos suficiente información para determinar el papel tan influyente e importante de Fernando II.

PALABRAS CLAVE: BIOGRAFÍA, Fernando II, Historia de la Cultura 


\title{
FERDINAND II OF THE HOLY ROMAN EMPIRE
}

\begin{abstract}
The following entry will develop an important aspect of the life of the Emperor of The Holy Roman Empire, Ferdinand II. We were deeply interested on this aspect of his biography because it includes transcendental themes such as the Thirty Years War. Citing exceptional writers, who decided to focus their articles and books mostly on the mentioned war, we collected sufficient information in order to determine the role as influential and important of Ferdinand II.
\end{abstract}

KEY WORDS: Biography, Fernando II, Cultural history

\section{Fernando II del Sacro Imperio Romano Germánico}

El siguiente trabajo relatará principalmente la importancia e influencia del emperador Romano Germánico, Fernando II. Iniciaremos este breve relato haciendo una introducción de la importante y reinante Dinastía Habsburgo de la cual Fernando II formó parte.

\section{Dinastía Habsburgo}

Sabiendo que Fernando II fue una persona muy influyente en su estadía en el trono como Emperador del Sacro Imperio Romano Germánico, este trabajo se enfocará principalmente en desarrollar y resaltar su rol en la Guerra de los Treinta Años.

Como bien lo expresa Pavel Marek en su texto La Dinastía Española y Papal en la Corte Imperial de Fernando II, la relación entre los miembros de la Casa Habsburgo llevó a éstos a ser lo que fueron. Felipe II en su reinado en España, consideró necesaria la intervención tanto del Emperador como del Rey para lograr de una u otra manera la estabilidad religiosa y económica de aquel momento. Notando la falta de decisión que tenían sus familiares en Viena, mandó a instalar una corte con la finalidad de convencerlos de adentrarse más decididamente en la política imperial de 
España. "Las situaciones en ambas ramas de esta casa eran muy tensas", afirma el referido autor. (Marek, 2008, p. 113-114)

\subsection{Fernando II y su ascenso al trono}

Una vez teniendo casi por completo la voluntad del Emperador para apoyar al Rey Español y así proteger a toda costa la Santa Sede, los protestantes empiezan a ganar batallas a su vez. El papa de aquel momento, al aliarse con Francia, inspiró cierta inseguridad e incertidumbre, que considero movió a los protestantes a, valga la redundancia, protestar. (Marek, 2008, p. 115) Según Miguel Lasso de La Vega y López de Tejada, (La embajada en Alemania del conde de Oñate y la elección de Fernando II. Madrid, 1929.) no es sino hasta que asciende Fernando de Estiria como Rey de Bohemia siendo la manera en la que, según Marek, "(...)los diplomáticos españoles y papales de la corte vienesa actuaron con consistencia(...)" imponiendo su elección. (Marek, 2008, p. 117)

En concordancia con el escritor Lasso de la Vega y López de Tejada, considero que Marek afirma lo anteriormente expuesto debido se sabía que el posteriormente conocido como Fernando II, defendería a capa y espada la religión católica; a fin de cuentas, éste era el interés principal de la Corte y es por esto, entre otras cosas, que velaron por su elección. (Lasso de la Vega y López de Tejada, 1929)

\subsection{Biografía de Fernando II}

Adentrándonos en materia, Fernando II fue un miembro activo de la Casa Habsburgo, hijo de Charles II y María Anna de Bavaria. Con ayuda de sus familiares ascendió a ser Rey de Bohemia y, por su apego a la religión Católica, al respirar aires protestantes en sus tierras decidió dar inicio a la rebelión, sucediendo en un principio en la ciudad de Bohemia. (Sturmberger, 2013) 
Por otro lado, el mismo autor, basándose en los textos de Robert Bireley y Cicely Veronica Wedgwood, ambos titulados The Thirty Years War, se describe a Fernando II de una manera mucho más detallada. Señalan que era una persona bondadosa, benevolente, un monarca imponente, completamente entregado a la creencia de la grandeza de su dinastía. Hablaba alemán, italiano, francés y español, por lo que deducimos tuvo una educación bastante completa. Tenía una pasión particular por la caza, libros religiosos, entre otras cosas. Basaba su política principalmente en principios religiosos, no obstante, más adelante veremos como esos principios dejaban de ser lo más importante en distintas ocasiones. Lo definen como un hombre indeciso y dependiente de los consejos de sus confesores jesuitas, no obstante, discrepo en afirmar esto citando por ejemplo a Martín Spahn, quien define a Fernando II como una persona que actuaba a conveniencia y con mucha rigidez. Tanto es así, que el mismo autor que afirma que dicho emperador era un hombre indeciso, en el desarrollo de su trabajo, nos damos cuenta como afirma que adentrándose en la guerra mostró mucha firmeza en cuanto a la toma de decisiones - por ejemplo, enlistando nuevamente a Wallenstein cuando todos querían lo contrario-. Ejerció una importante e influyente participación en su época por su característica pasión por el apego a la religión y su estricta e inflexible política religiosa. (Sturmberger, 2013)

\section{El Protestantismo y la Guerra de los Treinta Años}

Peter H. Wilson, cataloga a la Guerra que estará por iniciar como una de las más horribles que ha vivido la historia. Manuel Alcavde Mengual narra de una manera muy interesante las causas de la Guerra de los Treinta Años, sin embargo, me enfocaré principalmente en resaltar la influencia y participación que Fernando II brindó. (Alcavde Mengual, S/F, p. 2-3)

Marín Spahn, en la Enciclopedia Católica, establece cómo el protestantismo en Europa fue un proceso con altibajos; con esto me refiero a que no podemos considerar que la causa fue una sola o unas pocas; por el contrario, se esperó a contar con ese caldo de cultivo necesario para que fermentara en él el ambiente necesario para desencadenar una guerra que puede haber iniciado por problemas religiosos, sin embargo, el motivo 
de la guerra dejará de ser uno sólo a medida que pase el tiempo. No obstante, establece que el factor netamente clave para el desencadenamiento de esta guerra fue el protestantismo.

Por otro lado, autores afirman que definitivamente, la forma en que prohibieron el protestantismo en los lugares donde reinó la Dinastía, hizo que sus habitantes se revelaran y surgieran diversos disturbios en los cuales no nos centraremos ahora, sin intenciones de restar su importancia e influencia. (Spahn, 1999) (Wilson, 2009) Tanto es así, que Burkhardt, en su monografía, la Guerra de los Treinta Años intenta contrastar hasta qué punto podemos considerar la mencionada Guerra como la mayor acumulación de conflictos bélicos de la historia. (Gotthard, 2001, p.152) por lo que, si desarrollamos este trabajo resaltando los aspectos importantes de la guerra, nos desviaremos de nuestro objetivo principal, Fernando II.

Spahn afirma que "la lucha de la nobleza contra la dinastía alcanzó su punto más alto durante la última década del reino de Rodolfo II (1576-1612)." Ubicándonos en contexto, sabemos que este punto más alto al que se hace referencia, fue un poco antes de que Fernando II entrara al poder, inicialmente como Rey de Bohemia y posteriormente como Emperador del Sacro Imperio Romano Germánico. (Spahn, 1999) Es impactante como pocos autores definen la trascendencia del paso de ser Rey a ser Emperador, no obstante, Spahn, se encarga de enaltecer dicha ascendencia haciendo entender que esto, de una u otra manera, aseguraría la "dignidad imperial de su familia."

Es importante resaltar que en aquel momento no todos los católicos estaban en un mismo bando, y esto, entre otras cosas, hacía un poco más complicado la posibilidad de unificación para así formar un Imperio tan imponente como por ejemplo, el romano. (Vallejo Mejía, 2006, p. 58-59-60).

Sabiendo que Fernando II, no fue una persona querida en Bohemia, entre otras cosas, por su apego a la religión católica -también hay quienes opinan que sus decisiones fueron un tanto radicales- debemos saber que este desprecio causó distintas situaciones importantes e influyentes en la vida de Fernando II, como por ejemplo "La 
Segunda Defenestración de Praga", constituyendo un desafío para él seguir luchando. (Vallejo Mejía, 2006, p. 58)

\subsection{Situación Económica, un factor determinante para iniciar la Guerra}

Estando el emperador completamente seguro de que pretendía seguir, asumió el hecho de que la dinastía no estaba lista aún para la guerra, y lo podemos observar en el texto de Spahn, y en contraste con lo que Peter Wilson afirma alegando que la situación económica de la época no era fácil de llevar, corroborando esto, a su vez, con lo que afirma Pavel Marek, concluyendo que era necesario y determinante tener gran capital para afrontar la situación. Fernando II estaba consciente que se necesitaría mucho dinero y un ejército muy grande para salir vencedores de los enfrentamientos que debían llevar a cabo; por esto, esperó el momento preciso.

No fue sino hasta que recibió la ayuda de Maximiliano de Baviera, personaje poseedor del ejército más grande en todo el Imperio, de España y Portugal, quienes a su vez enviaron tropas, entre otras ayudas que impulsaron a Fernando a iniciar y dar el paso de erradicar al partido protestante. (Spahn, 1999).

\section{Unidos Maximiliano y Fernando II por una misma causa}

Según Spahn, "Maximiliano aterrorizó de tal manera al partido protestante, el cual había formado la Unión desde 1608, que fue completamente desmantelado." Luego de la primera batalla, de la cual salieron victoriosos, Fernando toma posesión de los territorios, empezando a expandir su imperio. (Spahn, 2009) En este punto, nos encontraríamos en un momento en el que lo que inició como una pequeña confrontación en Bohemia, se estará convirtiendo en una de las Guerras de más trascendencia de la historia. 


\subsection{Ansias de triunfar}

Es interesante observar como en un movimiento iniciado, en principio, por las ansias de erradicar el protestantismo, Fernando, no se mantuvo siempre en un mismo lado. (Del lado de aquellos que querían erradicar el protestantismo). No me refiero a esto afirmando que el emperador fuese de una u otra manera traidor a su causa, pero si pienso que de una manera muy inteligente, asimilando que la extensión de la guerra era inminente, manteniendo siempre su "target" muy presente, supo manejar la situación y aceptando el hecho de tener que incluir en su movimiento cualquier tipo de persona, bohemio, protestante, etc. Spahn, una vez más nos provee el ejemplo de esto, se trata de "Wallenstein, un noble bohemio, y el más hábil de todos los líderes de los mercenarios(...)" propuso mantener de la misma manera que sus enemigos, un ejército más grande y más fuerte. Confiando en esto, Fernando lo nombró general, a sabiendas de que estaba agregando a su causa una persona del otro bando. Por otro lado, es importante citar a Pavel Marek, cuando establece que el ejército de Wallenstein estaba compuesto por protestantes y católicos. En un momento dado, posterior- Fernando II hasta se prestó a agregar un luterano en su causa. (Spahn, 2009) Estos son ejemplos claros de la perspicacia de Fernando II; nos podemos dar cuenta de la fijación que tenía con ganar la guerra y consiguiéndolo ganando las batallas venideras, haciendo lo que fuese necesario para lograrlo. (Sturmberger, 2001)

Sucede algo curioso, debemos fijarnos cómo esta persona que añadieron al ejército del Emperador, sin ser un Habsburgo y sin compartir necesariamente todos los principios por los que se regía el Imperio, dirigió tantas batallas ganando aún más territorio. Es aún más interesante observar que Fernando II, persona que únicamente consideraba realmente capaz de coordinar eventos de los cuales se obtuviera un resultado próspero a Maximiliano, estaba orgulloso de su decisión.

La dinastía de los Habsburgo ganó tanto respeto que hasta aquellos que no eran de la Casa Habsburgo, sino protegidos por ellos, eran respetados. (Spahn, 1999) 


\section{El imperio de Fernando II de Habsburgo}

Pienso que es importante resaltar que, como muchos autores afirman, el Emperador Fernando II, de una u otra manera, se aprovechó del poder que tenía en sus manos y en muchas ocasiones, sin el consentimiento del pueblo, "revivió otros privilegios imperiales que habían caído en desuso" (Spahn, 1999), generando una revelación, fundamentada, desde mi punto de vista, por parte de la población que no necesariamente era protestante.

Haciendo referencia a la unidad con la que contaba el Imperio Romano, con sus excepciones, me permitiré realizar un contraste con respecto a este Imperio. Fernando II tenía tanta influencia y tanto poder de acción, que su pueblo, sin él imponerse, y siendo pueblo desde casi 50 años antes de que él entrara al poder, estaba consciente de que teniéndolo a él en el trono como emperador, dependerían del Imperio únicamente para asuntos muy específicos afirmando y asimilando que de una u otra manera las decisiones del Emperador serán las que los afectarían directamente, positiva o negativamente. (Vallejo Mejía, 2006)

Después de un tiempo de guerra, sin adentrarnos en la trascendencia de cada batalla, nos adelantaremos hasta el momento en el que el nuncio Carlos Caffa, con la bendición de Wallenstein,-referido general de tropas del Emperador- le solicitaba llevar la guerra a su fin en el Sacro Imperio, para pasar a concentrarse en problemas venideros con los turcos. (Marek, 2008)

El escenario en este momento era incierto para todos menos para Fernando II, era una persona con metas claras. El Imperio en general consideraba que el norte por el cual luchaban en principio todos juntos, se había perdido fundamentando esta afirmación, entre otras cosas, en que el Emperador añadía cualquier tipo de persona en el movimiento. (Sturmberger, 2013)

Este síntoma de tensión entre el emperador y los demás estados del imperio serán inminentes. Estaban inconformes, principalmente, con Wallenstein, solicitando su despido. (Spahn, 2009)-(Marek, 2008) Según Sturmberger, este despido fue conseguido en primera instancia; poco después el Emperador se dio cuenta de la 
necesidad de reincorporarlo. Con el descontento de los más importantes personajes de aquella época, nuevamente, se vio en la obligación de despedirlo y asesinarlo. Así, notamos una vez más, cómo Fernando II actuaba a conveniencia.

Posteriormente, con las ansias de mantener a su familia en el trono, luego de completar la reorganización de sus territorios -lo que nuevamente afirmaría el poder y la influencia que tenía frente al Imperio-, con el ascenso de su hijo al trono como Rey de los Romanos. (Spahn, 2009)

Citando a la reseña realizada por Sturmberger utilizando el libro de Cicely Veronica Wedwood -referido anteriormente- como base, concluiremos que los contemporáneos a su época, consideraban a Fernando como un "santo hecho monarca" (Sturmberger, 2013) y sus oponentes lo tildaban de tirano.

Estos escritores citados en el párrafo anterior consideran que quienes se encargaban de hacer historia en la época Romana-Católica del siglo XIX enaltecen al personaje y consideran que su papel fue completamente determinante; mientras que los historiadores liberales subestiman su importancia. Los historiadores actuales consideran que se debe reconocer a Fernando la gran importancia de convertir las provincias de Austria en un solo todo.

Para finalizar, me gustaría dejar abierta una afirmación que realiza el escritor Axel Gotthard alegando que Fernando II, para muchos, fue "Tan corrupto en su conciencia jurídica como el propio Wallenstein." (Gotthard, 2001: Pág. 153) 


\section{Bibliografía}

Alex Gotthart. (2009). El sacro imperio durante la Guerra de los Treinta Años. Consultado el 22 de junio de 2013. Disponible en: http://dialnet.unirioja.es/servlet/articulo?codigo $=1314711$

Cicely Veronica Wedgwood. (2005). The Thirty Years War. Consultado el 22 de Junio de 2013. Disponible en: http://books.google.co.ve/books?id=00TyV70CKAC\&dq=cicely+veronica+wedgwood ++thirty+years+war\&hl=es419\&sa $=$ X\&ei $=5$ XzRUe3oPJTW8gSj9YCQCg\&ved=0CCWQ6AEWAA

Hans Sturmberger. (2013). Ferdinand II. Consultado el 23 de junio de 2013. Disponible en: http://www.britannica.com/EBchecked/topic/204463/Ferdinand-II

Martín Spahn. 2013. La guerra de los treinta años. Consultado el 22 de junio de 2013. Disponible en: http://ec.aciprensa.com/g/guerratreinta.htm

Miguel Lasso de la Vega y López de Tejada (1929) La embajada en Alemania del conde de Oñate y la elección de Fernando II. Madrid, España. Consultado el 22 de Junio de 2013. Disponible en http://books.google.co.ve/books?id=00iEXwAACAAJ

Pablo Vallejo Mejía. 2006. Historia de las Relaciones Internacionales. Editorial Fondo Editorial Universidad Eafit. Disponible en: http://books.google.co.ve/books?id=KGX9HLImgC\&pg=PA49\&lpg=PA49\&dq=Fernando+II+de+Habsburgo\&source=bl\&ots=gaVdQ egyBY\&sig=KrXrk9JocQYvGV5QdJKKZfuBbmw\&hl=es\&sa=X\&ei=GfXJUZWyN4Hk8gTwl 4CQCw\&ved $=0 C E w Q 6 A E w B j g U \# v=$ onepage $\& q \& f=$ false

Pavel Marek. (2008). La diplomacia Española y la papal en la corte imperial de Fernando II. Universidad de "eské Budie jovice. Consultado el 22 de junio de 2013. Disponible en: http://campus.usal.es/ revistas_trabajo/index.php/Studia_Historica/article/viewFile/14 52/1519

Peter H. Wilson. (2009). The Thirty Years War. Europe's Tragedy.

Robert Birely. (2003). The Jesuits and The Thirty Years War. Consultado el 23 de Junio de 2013.2 Disponible en: http://books.google.co.ve/books?id=RL9YIUfckkAC\&printsec=frontcover\&dq=rOBERT + BIRELEY\&hl=es$419 \& \mathrm{sa}=X \& \mathrm{ei}=S H 3 R U b X J C Z L m 8 w T P t Y G w C w \& v e d=0 C D g Q 6 A E w A Q \# v=$ onepage $\& q=r$ OBERT\%20BIRELEY\&f=false 


\section{ANDREA CRISTINA REGLÁ CARRILLO}

Dirección: Las Mercedes, Calle La Cinta, Residencias Curiana, Apto 1-C, Caracas, Venezuela.

Teléfonos: 0414-790-7202 / 0212-9928735

E-mail: andreareglac23@gmail.com

Lugar de Nacimiento: La Asunción, Edo. Nueva Esparta, Venezuela.

Cedula de Identidad: V- 20.904.058

Fecha de Nacimiento: 23 de Julio de 1992

Edad: 21 años.

Estado Civil: Soltera.

Idiomas: Español, Inglés (Avanzado), catalán (Medio)

Licenciada en Estudios Liberales I Estudios Liberales y Derecho (Ambas carreras) Universidad Metropolitana de Caracas. Venezuela

\section{MARÍA ELENA DEL VALLE MEJÍAS}

María Elena Del Valle de Villalba, nacida en Caracas, Venezuela, el 10 de marzo de 1970, graduada de Profesora en la Universidad Pedagógica Libertador en el año 1992, con especialización en Historia Económica y Social de Venezuela en la Universidad Santa María, con Maestría en Historia Económica y Social de Venezuela, tesis con mención publicación y suma cum laude, Doctora en Ciencias de la Educación en el Pedagógico de Caracas, Coordinadora del Núcleo de Investigaciones Geohistóricas Lisandro Alvarado, cuenta con proyectos financiados por la UPEL y con participación de la Universidad Complutense de Madrid, con proyectos financiados por el FONACIT, investigadora certificada de la Universidad Complutense de Madrid, el grupo internacional CONCILIUM y del CELARG, ponente regular en eventos nacionales e internacionales, adscrita a la línea de investigación, Ciencias del lenguaje y con siete años de investigación en el área de análisis del discurso. Profesora Ordinaria de la Universidad Pedagógica Libertador. Investigadora acreditada del CYTED, la AECID, profesora de la UNIMET con postdoctorado en semiótica y pragmática. 